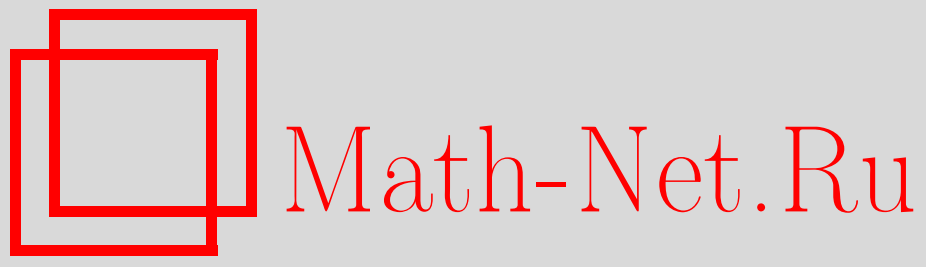

В. Ю. Калошин, Проблема Гильберта-Арнольда и оценка цикличности полициклов на плоскости и в пространстве, Функи. анализ и его прил., 2001, том 35, выпуск 2, 78-81

DOI: https://doi.org/10.4213/faa249

Использование Общероссийского математического портала MathNet.Ru подразумевает, что вы прочитали и согласны с пользовательским соглашением

http://www.mathnet.ru/rus/agreement

Параметры загрузки:

IP : 54.237 .59 .107

26 апреля 2023 г., $17: 27: 20$

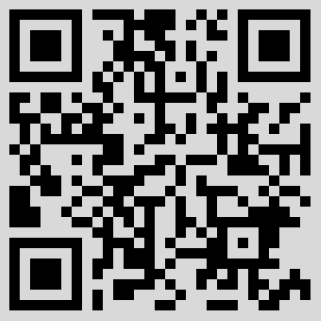


взаимно однозначно (и, значит, взаимно непрерывно) отображал $\mathscr{H}(E)$ на пространство $l_{2}\left\{b_{k}\right\}$ последовательностей $c:=\left\{c_{k}\right\}_{-\infty}^{+\infty}$ с нормой

$$
\|c\|^{2}=\sum_{-\infty}^{+\infty}\left|c_{k}\right|^{2} b_{k}<\infty .
$$

Последовательности $\Lambda$, описание которых дается теоремой 6 , назовем интерполяционными. Имеет место

Теорема 7. Если некоторая последовательность $\Lambda=\left\{\lambda_{k}\right\}_{-\infty}^{+\infty}, \inf _{k} \operatorname{Im} \lambda_{k}>0$, порождает взаимно однозначное отображение $\mathscr{J}_{\Lambda}$ регулярного пространства $\mathscr{H}(E)$ на пространство $l_{2}\left\{b_{k}\right\}$, то верны двусторонние оценки

$$
b_{k} \asymp \operatorname{Im} \lambda_{k} e^{-2 \omega \operatorname{Im} \lambda_{k}}\left|E\left(\lambda_{k}\right)\right|^{-2}, \quad k \in \mathbb{Z} .
$$

Далее, оператор $\mathscr{J}_{\Lambda}$ является изоморфизмом регулярного пространства $\mathscr{H}(E)$ на $l_{2}\left\{b_{k}\right\}$ с весом, удовлетворяющим условию (7), тогда и только тогда, когда последовательность $\Lambda$ интерполяционная. Единственное решение интерполяционной задачи $f\left(\lambda_{k}\right)=c_{k}\left(\lambda_{k} \in \Lambda\right)$ дается рядом (6), который сходится как по норме пространства $\mathscr{H}(E)$, так и равномерно на компактах плоскости.

Выражаю глубокую признательность Д. 3. Арову за многочисленные обсуждения результатов статьи.

\title{
ЛИТЕРАТУРА
}

1. Губреев Г. М. Функц. анализ и его прил., 33, вып. 1, $62-65$ (1999). 2. Губреев Г. М. Матем. сб́., 190, № 12 (1999). 3. De-Branges L. Hilbert spaces of entire functions. Prentice Hall, 1968. 4. Сахнович Л. А. Алгебра и анализ, 5, вып. 1. 3-80 (1993). 5. Гарнетт Дж. Ограниченные аналитические функции. Мир, М., 1984. 6. Павлов Б. С. ДАН СССР, 247, № 1, 37-40 (1979). 7. Джрбашян М. М. Изв. АН АрмССР, Математика, 19, № 2 (1984).

Южноукраинский педагогический госуниверситет, г. Одесса

Поступило в редакцию 9 декабря 1999 г.

УДК 517.938

\section{Проблема Гильберта-Арнольда и оценка цикличности полициклов на плоскости и в пространстве}

\author{
(c) 2001. В. Ю. КАлошин
}

1. Полициклы на плоскости. Мотивировкой для следующего определения является проблема Гильберта-Арнольда о равномерной ограниченности числа предельных циклов для типичных семейств гладких векторных полей на сфере $S^{2}$ (см., например, $[1,6]$ ).

ОПРЕДЕЛЕНИЕ 1. Полицикл $\gamma$ векторного поля $\dot{x}=v\left(x, \varepsilon^{*}\right), x \in S^{2}, \varepsilon^{*} \in B,-$ это циклически упорядоченный набор положений равновесия $p_{1}, \ldots, p_{k}$ (возможно, с повторениями) и набор фазовых кривых $\gamma_{1}, \ldots, \gamma_{k}$ (без повторений), их 
соединяющих, т. е. $j$-я кривая $\gamma_{j}$ соединяет $p_{j}$ с $p_{j+1}$, где $B$ - область евклидова пространства.

Цикличность полицикла $\gamma-$ это минимальное число $\mu(\gamma)$, для которого найдутся окрестность $U$ полицикла $\gamma \subset U \subseteq S^{2}$ и окрестность $V$ значения параметра $\varepsilon^{*} \in V \subseteq B$, обладающие следующим свойством: любое поле, соответствующее $\varepsilon \in V$, имеет не более $\mu(\gamma)$ сосуществующих циклов в $U$, и хаусдорфово расстояние между каждым из этих циклов и полициклом $\gamma$ стремится к 0 при $\varepsilon \rightarrow \varepsilon^{*}$.

Положение равновесия называется элементарным, если линеаризация поля имеет не более одного нулевого собственного значения. Полицикл, имеющий только элементарные положения равновесия, называется элементарным.

Элементарное число изикличности $E(n)$ - это максимальная цикличность элементарного полицикла, встречающегося в типичном $n$-параметрическом семействе $C^{\infty}$-гладких векторных полей на сфере $S^{2}$.

ТЕОРема 1. Для любого положительного числа $n$ әлементарное число изикличности допускает оценку $E(n) \leqslant 2^{25 n^{2}}$.

В [6] показано, что $E(n)<\infty$.

2. Бифуркации полициклов в пространстве. Определение 1 полицикла дословно переносится на многомерный случай. Рассуждения, аналогичные приведенным в [6, p. 3], показывают, что набор из бесконечного числа предельных циклов равномерно ограниченной длины, лежащих в компактном множестве, имеет предельный полицикл. Приведем классический пример полицикла в пространстве.

2.1. Полицикл Шильникова. Полицикл Шильникова - это полицикл, встречающийся в типичном 1-параметрическом семействе векторных полей в $\mathbb{R}^{3}$ и состоящий из седло-фокуса $p$ с собственными значениями $\lambda>0, \mu \pm i \omega, \mu<0$, $\lambda+\mu>0$, и гомоклинической траектории $\gamma=W^{u}(p) \cap W^{s}(p)$.

Рассмотрим сечение Пуанкаре $T$, трансверсально пересекающее фазовую кривую $\gamma \backslash\{p\}$. Для открытого подмножества $U \subset T$ определено отображение Пуанкаре $\Delta: U \rightarrow T$. В отличие от двумерного случая, $\Delta$ может иметь не только неподвижные точки, но и точки периода, большего, чем 1. Анализ топологии отображения Пуанкаре $\Delta$ показывает, что оно имеет счетное число сосуществующих подков Смейла $[4,9]$. Можно показать, что для любого $k \in \mathbb{Z}$ число периодических точек отображения $\Delta$ периода $k$ бесконечно. Одна из причин этого - комплексно-сопряженные собственные значения.

2.2. Оценка цикличности многомерного полицикла. Пусть $\gamma \subset \mathbb{R}^{N}-$ полицикл векторного поля $\dot{x}=v\left(x, \varepsilon^{*}\right), x \in \mathbb{R}^{N}, \varepsilon^{*} \in B$. Тогда $\gamma$ можно представить в виде объединения положений равновесия $\left\{p_{j}\right\}_{j \in J}$ и соединяющих их фазовых кривых $\left\{\gamma_{j}\right\}_{j \in J}$. Трубчатая окрестность $T_{\gamma}$ полицикла $\gamma$ - это объединение окрестностей $\left\{U_{j}\right\}_{j \in J}$, положений равновесия $\left\{p_{j}\right\}_{j \in J}$ и трубчатых окрестностей $\left\{T_{j}\right\}_{j \in J}$ фазовых кривых $\left\{\gamma_{j}\right\}_{j \in J}$.

ОПРЕДЕЛЕНИЕ 2. $k$-цһикличность полицикла $\gamma-$ это минимальное число $\mu(\gamma, k)$, для которого найдутся трубчатая окрестность $\gamma \subset T_{\gamma} \subset \mathbb{R}^{N}$, окрестность $V$ значения параметра $\varepsilon^{*} \in V \subseteq B$ и сечения Пуанкаре (гиперповерхности) $T_{j}$, трансверсально пересекающие фазовые кривые $\gamma_{j}$ в одной точке для каждого $j \in J$. Кроме того должно выполняться следующее условие: любое поле, соответствующее $\varepsilon \in V$, имеет не более $\mu(\gamma, k)$ сосуществующих циклов в $T_{\gamma}$, каждый 
из которых пересекает каждое сечение $T_{j}$ ровно в $k$ различных точках. Каждый из таких циклов называется $k$-обходньıм изиклом или $k$-цјиклом.

Положение равновесия называется квазиэлементарным, если матрица линеаризации имеет только вещественные собственные значения и они либо нерезонансны, либо сильно однорезонансны, либо существует одно нулевое с показателем Лоясевича 1 , а остальные нерезонансны и попарно различны ${ }^{1)}$. Полицикл, имеющий только квазиэлементарные положения равновесия, называется квазиэлементарным.

Квазиэлементарное число $k$-циикличности QЕ $(N, n, k)$ - это максимально возможная $k$-цикличность квазиэлементарного полицикла, встречающегося в типичном $n$-параметрическом семействе $C^{\infty}$-гладких векторных полей в $\mathbb{R}^{N}$.

Теорема 2. Для любых положительных $N$ (размерности фазового пространства), $n$ (числа параметров) и $k$ («обходности») квазиэлементарное число ичикличности допускает оценку $\mathrm{QE}(N, n, k) \leqslant 2^{T^{2}}$, где $T=6 N n k$.

2.3. Кратность ростков типичных отображений. Под $n$-типичными $C^{m}$-гладкими ростками понимаются ростки, которые встречаются у типичного глобального $C^{m}$-гладкого отображения $f: \mathbb{R}^{n} \rightarrow \mathbb{R}^{N}$. Например, при $n=N=2$ по теореме Уитни о типичных отображениях 2-мерных многообразий возможны 3 типа 2-типичных ростков: 1-1, складка и сборка [2].

ОПРЕДЕЛЕНИЕ 3 . Пусть $f:\left(\mathbb{R}^{n}, 0\right) \rightarrow\left(\mathbb{R}^{n}, 0\right)$ есть $C^{m}$-гладкий росток. Его геометрической кратностью называется максимальное число прообразов в окрестности нуля $G_{0}(f)=\sup _{r \rightarrow 0+} \sup _{y \in \mathbb{R}^{n}} \#\left\{|x|<r: x \in \phi^{-1}(y)\right\}$, где $\phi-$ какой-то представитель ростка $f ; G_{0}(f)$ не зависит от выбора представителя $\phi$.

Теорема 3. Для $n$-типичного $C^{n+1}$-гладкого ростка $f:\left(\mathbb{R}^{n}, 0\right) \rightarrow\left(\mathbb{R}^{n}, 0\right)$ выполнена оценка $G_{0}(f) \leqslant 2^{n(n-1) / 2} n^{n}$.

Можно также оценить геометрическую кратность композиции $P \circ j^{m} f:\left(\mathbb{R}^{n}, 0\right)$ $\rightarrow\left(\mathbb{R}^{n}, 0\right)$, где $f:\left(\mathbb{R}^{n}, 0\right) \rightarrow\left(\mathbb{R}^{N}, 0\right)$ есть $n$-типичный $C^{n+1}$-гладкий росток, а $P: J^{m}\left(\mathbb{R}^{n}, \mathbb{R}^{N}\right) \rightarrow\left(\mathbb{R}^{n}, 0\right)$ - полином известной степени $d$, имеющий регулярные точки. Для композиции $P \circ j^{m} f$ выполнена оценка $G_{0}\left(P \circ j^{m} f\right) \leqslant 2^{n(n-1) / 2}(d n)^{n}$, не зависящая от размерности промежуточного пространства $J^{m}\left(\mathbb{R}^{n}, \mathbb{R}^{N}\right)$. Эта оценка применяется при доказательстве теорем 1 и 2.

Доказательство сформулированных выше теорем использует теорию нормальных форм $[5,6]$, метод Хованского $[6,8]$ и некоторые новые факты из теории стратифицированных множеств, сформулированные в [7]. При доказательстве теоремы 2 используется также специальная мультиструйная теорема трансверсальности [3].

\section{ЛИТЕРАТУРА}

1. Арнольд В. И., Аносов Д. В. и др. Динамические системы - I, Итоги науки и техники. Современные проблемы математики. Фундаментальные направления, ВИНИТИ, М., 1985. 2. Арнольд В. И., Варченко А. Н., Гусейн-Заде С. М. Особенности дифференцируемых отображений. Т. 1, Наука, 1982. 3. Grigoriev A., Yakovenko S. J. Diff. Equations, 150, No. 2, 349-362 (1998). 4. Guckenheimer J., Holmes P. Nonlinear Oscillations, Dynamical Systems, and Bifurcation of Vector Fields. Appl. Math. Sci., Vol. 42. Springer-Verlag, New York-Berlin, 1983. 5. Ильяшенко Ю. С., Яковенко С. Ю. УМН, 46, вып. 1 (277), 3-39

1) Определения нерезонансных и сильно однорезонансных собственных значений см., например, в $[5,6]$. 
(1991). 6. Ilyashenko Yu., Yakovenko S. Amer. Math. Soc. Transl. Ser. 2, Vol. 165, Amer. Math. Soc., Providence, RI, 1995, pp. 1-20, 21-95. 7. Ilyashenko Yu., Kaloshin V. In: Proceedings of the Arnoldfest. Fields Inst. Comm., Vol. 24, Amer. Math. Soc, 1999, pp. 241271. 8. Хованский А. Г. Малочлены. Библиотека математика, вып. 2, Фазис, М., 1997. 9. Шильников Л. П. ДАН СССР, 160, 558-561 (1965).

Московский государственный университет

им. М. В. Ломоносова,

Поступило в редакцию механико-математический факультет

28 декабря 1998 г.

e-mail: kaloshin@math.princeton.edu

УДК 517.98

\title{
Об асимптотических гомоморфизмах в алгебры Калкина*
}

\author{
(c) 2001. В. М. МАНУЙЛОВ
}

Задача классификации расширений $C^{*}$-алгебр послужила стимулом проникновения топологических методов в теорию $C^{*}$-алгебр [3]. Как показано в [7], расширения ядерных $C^{*}$-алгебр описываются в терминах $K K$-функтора Каспарова, однако в общем случае пока подобного описания нет. В настоящей заметке мы определяем группу так называемых фантомных расширений (возможно, пустую) и в случае, когда $A$ является двукратной надстройкой, даем описание ее расширений в терминах $E$-теории Конна-Хигсона [5] и группы фантомных расширений.

Для $C^{*}$-алгебр $A$ и $B$ через $[[A, B]]$ мы обозначаем множество гомотопических классов асимптотических гомоморфизмов из $A$ в $B[5,6]$. Мы предполагаем, что $A$ сепарабельна, а $B$ имеет строго положительный элемент. Через $\operatorname{Ext}(A, B)$ мы обозначаем множество гомотопических классов гомоморфизмов из $A$ в алгебру Калкина $Q(B \otimes \mathscr{K})=M(B \otimes \mathscr{K}) / B \otimes \mathscr{K}$, где $\mathscr{K}$ есть $C^{*}$-алгебра компактных операторов, а $M$ обозначает алгебру мультипликаторов [13]. С помощью инварианта Басби [4] это множество отождествляется с множеством расширений алгебры $A$ посредством алгебры $B \otimes \mathscr{K}$. Аналогично, через $\operatorname{Ext}^{a s}(A, B)$ мы обозначаем множество гомотопических классов асимптотических гомоморфизмов алгебры $A$ в $Q(B \otimes \mathscr{K})$. (Асимптотические) гомоморфизмы $f_{0}, f_{1}: A \rightarrow Q(B \otimes \mathscr{K})$ называются гомотопными, если существует (асимптотический) гомоморфизм $F: A \rightarrow Q(B \otimes \mathscr{K} \otimes C[0,1])$, композиции которого с гомоморфизмами вычисления в 0 и 1 совпадают с $f_{0}$ и $f_{1}$ соответственно. Все указанные множества обладают естественной структурой абелевой группы в случае, когда $A$ является надстройкой, т.е. $A=S D=C_{0}(\mathbb{R}) \otimes D$ для некоторой $C^{*}$-алгебры $D$.

Так как любой настоящий гомоморфизм можно рассматривать как асимптотический, имеется естественное отображение

$$
i: \operatorname{Ext}(A, B) \rightarrow \operatorname{Ext}^{a s}(A, B) .
$$

Хотя типично асимптотических гомоморфизмов больше, чем настоящих, мы показываем, что отображение $i$ эпиморфно, если $A-$ надстройка. Заодно мы получим новое описание $E$-теории.

*Работа частично поддержана РФФИ (грант №99-01-01201). 